\title{
Stability of a Series of BODIPYs in Acidic Conditions: An Experimental and Computational Study into the Role of the Substituents at Boron
}

\author{
Maodie Wang, ${ }^{\dagger}$ M. Graça H. Vicente, ${ }^{\dagger}$ Deanna Mason, ${ }^{\ddagger}$ and Petia Bobadova-Parvanova* ${ }^{*}+\infty$ \\ ${ }^{\dagger}$ Department of Chemistry, Louisiana State University, Baton Rouge, Louisiana 70803, United States \\ ${ }^{\ddagger}$ Department of Chemistry, Rockhurst University, Kansas City, Missouri 64110, United States
}

\section{Supporting Information}

\begin{abstract}
Boron-dipyrromethene (BODIPY) dyes have been extensively investigated in recent years for a variety of bioanalytical and bioimaging applications. The success of these applications relies on the stability of BODIPYs, particularly under acidic conditions. In this work, the stability of a series of 4,4'-disubstituted BODIPYs ( $-\mathrm{F},-\mathrm{CN},-\mathrm{Ph},-\mathrm{Me},-\mathrm{OMe})$ toward addition of excess trifluoroacetic acid (TFA) was studied systematically and comprehensively through ${ }^{11} \mathrm{~B}$ and ${ }^{1} \mathrm{H}$ NMR, UV-vis, fluorescence, thin layer chromatography, mass spectrometry, and infrared. The results indicate that $4,4^{\prime}$ dicyano-BODIPY 2 is the most stable among this series and remains unchanged even 3 days after addition of excess TFA.
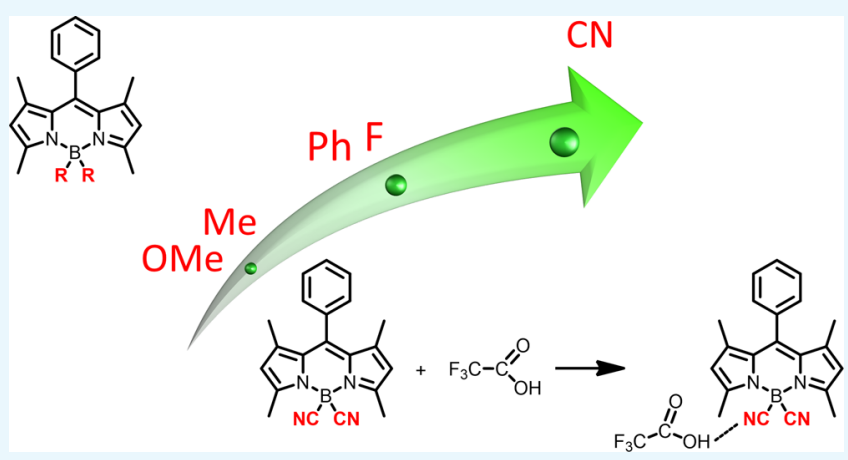
On the other hand, 4,4'-dimethyl-BODIPY 3 and 4,4' dimethoxy-BODIPY 5 are the least stable, toward addition of TFA, and the 4,4'-diphenyl and 4,4'-difluoro-BODIPYs 1 and 4 were found to have intermediate stability. The experimental analysis and comparison with theoretical calculations indicate that the 4,4'-dicyano-BODIPY 2 has the greater aromaticity of the series, as evaluated by the BLA parameter, decreased charge on boron, and upon TFA addition it forms an unusually stable BODIPY $2 \cdots$ TFA complex. On the other hand, all other BODIPYs decompose within hours after TFA addition. Computational modeling demonstrates that 4,4'-dicyano substitution increases aromaticity and stabilizes the $\mathrm{B}-\mathrm{N}$ bond, resulting in the most stable compound from the series studied.
\end{abstract}

\section{INTRODUCTION}

4,4-Difluoro-4-bora-3a,4a-diaza-s-indacene fluorophores (known as boron dipyrromethenes or BODIPYs) have many desirable photophysical properties for bioanalytical and bioimaging applications, including sharp absorption and emission bands in the visible spectral region, high fluorescence quantum yields, and high molar extinction coefficients. ${ }^{1,2}$ Compared to other commercially available fluorescent dyes (such as cyanines, rhodamines, and oxazines), BODIPY dyes generally display higher solubility, higher cell membrane permeability, lower cytotoxicity, and are photochemically more stable. ${ }^{3}$ Therefore, BODIPY dyes are finding multiple applications in biology and medicine, for example, in molecular imaging, biological labeling, and theragnostic. These applications often require the covalent attachment of fluorescent dyes to target groups or affinity ligands, such as peptides, antibodies, and nanoparticles, under strong acidic or basic conditions, and/ or high temperatures. ${ }^{4}$ As a result, many commercially available dyes, including some BODIPYs, are unstable to typical conjugation procedures and conditions, decomposing during the conjugation or purification processes, for example, during high-performance liquid chromatography using even a small percentage of trifluoroacetic acid (TFA) for elution, limiting their practical use for these applications. Therefore, we conducted a comprehensive study on the stability of a series of BODIPY dyes bearing different substitutions at boron, in the presence of excess TFA.

Although many BODIPYs functionalized at boron have been recently reported, including F-BODIPYs, ${ }^{1,5-9}$ Cl-BODIPYs, ${ }^{10-13}$ H-BODIPYs, ${ }^{14}$ C-BODIPYs, ${ }^{15-20}$ O-BODIPYs, ${ }^{21-26}$ OH-BODIPYs, ${ }^{27,28}$ and CN-BODIPYs, ${ }^{29-32}$ their stability and reactivity have not yet been comprehensively investigated. In 2011, Yan et al. ${ }^{33}$ reported that a 4,4'-diphenyl-BODIPY was more stable under both acidic (using di or trichloroacetic acid) and basic conditions (using ammonium hydroxide) than the corresponding 4,4' -difluoro, 4,4' ${ }^{\prime}$-dimethyl, and 4,4' -dimethoxyBODIPYs, using ${ }^{11} \mathrm{~B}$ NMR. More recently, Rumyantsev's group $^{34,35}$ studied the kinetic stability of a series of $4,4^{\prime}$ difluoro-BODIPYs in both aqueous and nonaqueous solutions and found a relationship between the dissociation rate with the solution acidity and the charge on nitrogen. We have recently reported $^{36}$ that cyano substitution at the boron atom significantly increases BODIPY stability under acidic conditions, particularly for the 4,4'-dicyano-BODIPYs. Interestingly, Ortiz's et al. ${ }^{17}$ have demonstrated that $4,4^{\prime}$-dicyano

Received: March 5, 2018

Accepted: $\quad$ May 3, 2018

Published: May 21, 2018 
Scheme 1. Synthesis of BODIPYs 1-5

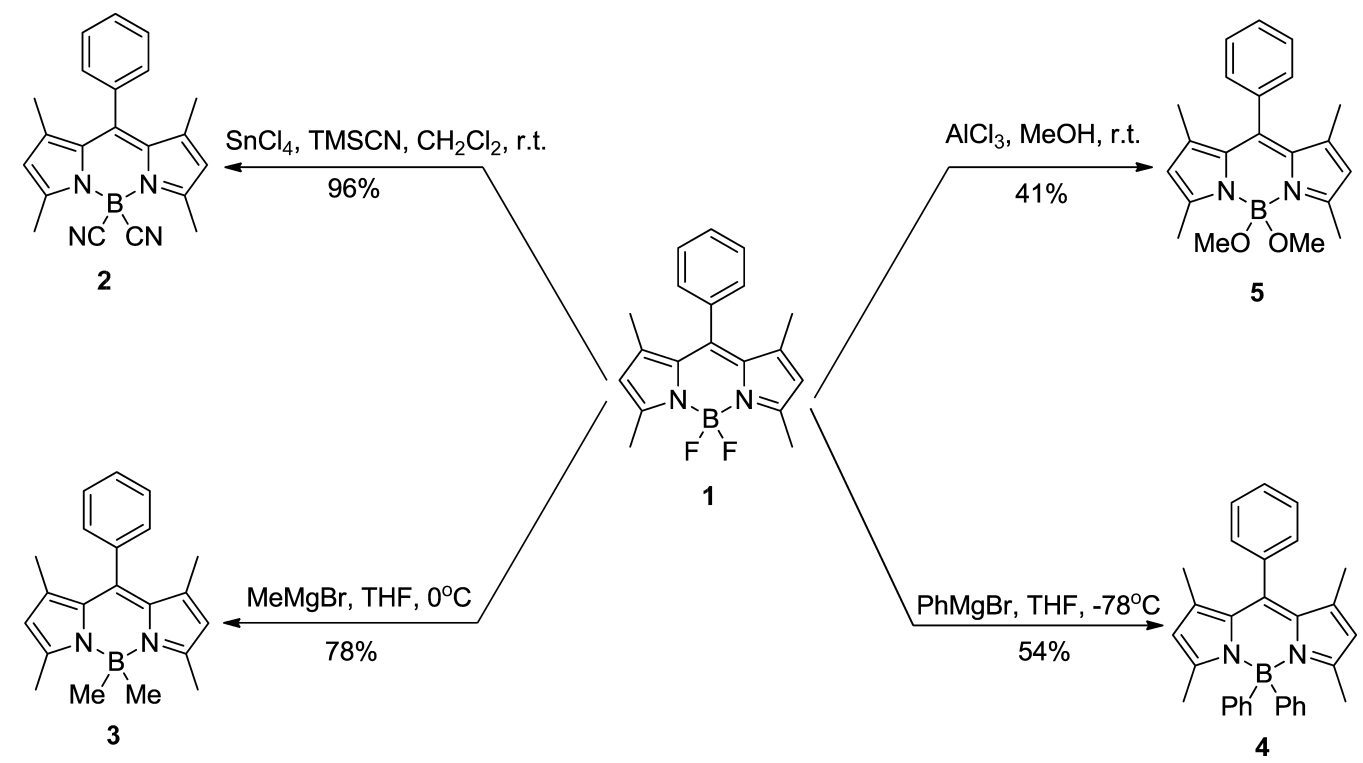

substitution also enhances the lasing stability of $4,4^{\prime}$-dicyanoBODIPYs in thin films.

Despite the spectacular increase on BODIPY literature in recent years, studies on BODIPY stabilities in aqueous and organic solutions have lagged behind, although these are important for further practical applications of this type of dye, especially the effects of substituents on boron on BODIPY stability. ${ }^{37}$ Herein, we report a comprehensive study on the stability of a series of 4,4'-disubstituted BODIPYs (Scheme 1) under strong acidic conditions. We used several experimental methods, including ${ }^{11} \mathrm{~B} \mathrm{NMR},{ }^{1} \mathrm{H} \mathrm{NMR}$, thin layer chromatography (TLC), UV-vis, fluorescence, mass spectrometry (MS), and infrared (IR), as well as computational modeling, to gain insights into the properties of the BODIPYs, aimed at identifying spectral characteristics and exploring the reasons behind the observed differences in stabilities. On the basis of existing previous research, ${ }^{3,33-35}$ we chose $4,4^{\prime}$-difluoro-1,3,5,7tetramethyl-8-phenyl-BODIPY $\mathbf{1}$ as the model compound and examined the effect of disubstitution at the boron atom, with cyano 2, methyl 3, phenyl 4, and methoxy 5 groups, on the stability under excess TFA in organic $\left(\mathrm{CHCl}_{3}\right.$ or $\left.\mathrm{CH}_{2} \mathrm{Cl}_{2}\right)$ solutions.

\section{RESULTS AND DISCUSSION}

The present study focuses on the investigation of BODIPY stability upon addition of TFA. All BODIPYs were analyzed in air at room temperature. If no TFA was added, all BODIPYs from the series studied showed no decomposition after 3 days in $\mathrm{CDCl}_{3}$ or $\mathrm{CH}_{2} \mathrm{Cl}_{2}$ solutions under air.

2.1. NMR Studies. The stability of BODIPYs $\mathbf{1 - 5}$ in $\mathrm{CDCl}_{3}$ solutions upon addition of 10 equiv of TFA was investigated by ${ }^{1} \mathrm{H}$ NMR and ${ }^{11} \mathrm{~B}$ NMR spectra over a period of 2 days $\left({ }^{1} \mathrm{H}\right.$ NMR) and 3 days $\left({ }^{11} \mathrm{~B}\right.$ NMR).

2.1.1. ${ }^{11} B N M R$. The ${ }^{11} \mathrm{~B}$ NMR spectra are shown in Figures S1-S5 of the Supporting Information. Different behaviors were observed for the different BODIPYs in this series. 4,4'-DifluoroBODIPY 1 was stable within the first $1 \mathrm{~h}$ but the characteristic triplet peak at 0.69 ppm gradually broadened over time, and additional peaks were observed upfield after $5 \mathrm{~h}$ of TFA addition (Figure S1). In contrast, the characteristic singlet in the ${ }^{11} \mathrm{~B}$ NMR spectrum of 4,4'-dicyano-BODIPY 2 observed at $-16.87 \mathrm{ppm}$, slightly shifts to $-16.60 \mathrm{ppm}$ upon addition of TFA and remains unchanged after that for up to the 3 day period investigated (Figure S2). We believe that this slight change in the chemical shift is due to the formation of a

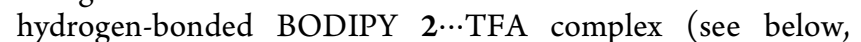
Sections 2.1.2, 2.3, and 2.6). On the other hand, the ${ }^{11} \mathrm{~B}$ NMR spectra for 4,4'-dimethyl-BODIPY 3 (Figure S3) and 4,4'-dimethoxy-BODIPY 5 (Figure S5) changed immediately upon addition of TFA. In both cases, the original singlet peaks at -0.78 and $2.57 \mathrm{ppm}$ for 3 and $\mathbf{5}$, respectively, immediately disappeared upon addition of TFA, indicating complete degradation of these BODIPYs. New peaks were observed in the ${ }^{11} \mathrm{~B}$ NMR spectra of 3 and 5 upon addition of TFA, downfield shifted in the case of $\mathbf{3}$ and upfield shifted in the case of 5 , indicating the formation of multiple boron degradation products. For 4,4'-diphenyl-BODIPY 4, the characteristic singlet at $-0.10 \mathrm{ppm}$ significantly broadened upon TFA addition, but it was still present after 3 days, and no noticeable new peaks were observed (Figure S4).

On the basis of the ${ }^{11} \mathrm{~B}$ NMR spectra results, BODIPY 2 is the most stable compound, remaining unchanged even 3 days after addition of 10 equiv of TFA. 4,4'-Difluoro-BODIPY 1 and 4,4'-diphenyl-BODIPY 4 exhibit intermediate stability, with some of the compound appearing to remain in the solution 3 days after addition of TFA, whereas 4,4'-dimethyl-BODIPY 3 and 4,4'-dimethoxy-BODIPY 5 are the least stable, decomposing immediately upon addition of TFA. This is in general agreement with previous stability studies of a series of 4-bora1,3,5,7-tetramethyl-2,6-diethyl-8-methyl-3a,4a-diaza-s-indacenes with 4,4'-difluoro, 4,4'-diphenyl, 4,4' -dimethyl, and 4,4'dimethoxy substitution. ${ }^{33}$ The $4,4^{\prime}$-diphenyl-BODIPY remained stable for 3 days upon addition of 50 equiv of dichloroacetic acid, whereas the $4,4^{\prime}$-difluoro analog partially decomposed after 1 day and the 4,4'-dimethyl analog completely decomposed over $6 \mathrm{~h}$. In the presence of 10 equiv of dichloroacetic acid, the 4,4'-dimethoxy-BODIPY decomposed over a period of $12 \mathrm{~h}$, giving the corresponding 4,4'-diacetate product. These BODIPYs completely decomposed within $4 \mathrm{~h}$ when TFA was used. Interestingly, the authors of this paper reported that the $4,4^{\prime}$-diphenyl-BODIPY was 
more stable than the corresponding 4,4'-difluoro compound under the acidic conditions. In the case of TFA, the $4,4^{\prime}$ difluoro-BODIPY decomposed within $3 \mathrm{~h}$, whereas the $4,4^{\prime}$ diphenyl-BODIPY remained stable for $4 \mathrm{~h}^{33}$

To further understand the observed NMR spectra and trends, the ${ }^{11} \mathrm{~B}$ NMR chemical shifts of all studied BODIPYs were calculated in chloroform using the density functional theory (DFT) method at the B3LYP/6-31+G(d,p) level (Table $1)$. In agreement with previous results, ${ }^{36}$ cyano substitution

Table 1. Experimentally Determined and B3LYP/6$31+G(d, p)$-Calculated ${ }^{11} \mathrm{~B}$ Chemical Shift $(\delta)$ in Chloroform Using $\mathrm{BF}_{3} \cdot \mathrm{OEt}_{2}$ as Reference, and NPA Atomic Charges on the Boron Atom $\left(q_{\mathrm{B}}\right)$ for the Series of BODIPYs Studied

\begin{tabular}{ccccc} 
BODIPY & boron substituent & ${ }^{11} \mathrm{~B} \delta_{\text {exp }}(\mathrm{ppm})$ & ${ }^{11} \mathrm{~B} \delta_{\text {calc }}(\mathrm{ppm})$ & $q_{\mathrm{B}}($ a.u. $)$ \\
$\mathbf{1}$ & $\mathrm{F}$ & 0.69 & 1.21 & 1.294 \\
$\mathbf{2}$ & $\mathrm{CN}$ & -16.87 & -17.24 & 0.484 \\
3 & $\mathrm{Me}$ & -0.78 & -2.39 & 0.769 \\
4 & $\mathrm{Ph}$ & -0.10 & -0.42 & 0.743 \\
$\mathbf{5}$ & $\mathrm{OMe}$ & 2.57 & 1.39 & 1.222 \\
\hline
\end{tabular}

results in a significant shift to negative $\delta$ values, which can be explained by the significant decrease in the charge on the boron atom, $q_{\mathrm{B}}$ (Table 1). A similar tendency can be observed for all other BODIPYs: the smaller the charge on the B atom, the greater the upfield shift. The agreement between calculated and observed values is good, within $0.5 \mathrm{ppm}$ for most BODIPYs, with the exception of $\mathbf{3}$ and $\mathbf{5}$, where the calculated shift differs from the experimental value by $1-1.5 \mathrm{ppm}$. Furthermore, the calculated ${ }^{11} \mathrm{~B}$ chemical shifts for the $4,4^{\prime}$-dicyano-BODIPY $2 \cdots$ TFA complexes bearing one or two TFA molecules hydrogen bonded to the nitrogen atoms of the $\mathrm{CN}$ groups are -17.95 and $-17.14 \mathrm{ppm}$, respectively. These results indicate that upon addition of TFA to BODIPY 2 , such complexes could form, as observed experimentally with a slight downfield shift in the boron chemical shift (Figure S2). These complexes are further discussed below in Sections 2.1.2, 2.3, and 2.6.

To gain insight about potential degradation products being formed upon addition of TFA in the case of BODIPYs 1, 3, 4, and 5 , the ${ }^{11} \mathrm{~B}$ NMR chemical shifts of a series of possible intermediates and products of the reaction of BODIPYs with TFA were calculated (Table S1, Supporting Information). Compounds of the type $\mathrm{BX}_{3}$ (trivalent boron) and $\mathrm{BX}_{4}$ (tetravalent boron) were chosen with the intent to cover a wide variety of possibilities. No peaks that could correspond to a trivalent boron compound were observed in the experimental spectra with the only exception of 4,4'-dimethyl-BODIPY 3, where a downfield peak around $55 \mathrm{ppm}$ was observed (Figure S3, Supporting Information), indicating a possible $\mathrm{BMe}_{2}\left(\mathrm{O}_{2} \mathrm{CCF}_{3}\right)$ product. On the other hand, multiple tetravalent compounds could explain the observed NMR spectra. For example, the tetravalent products that could be formed from addition of TFA to BODIPY 1, e.g., $\left[\mathrm{BF}_{2}\left(\mathrm{O}_{2} \mathrm{CCF}_{3}\right)_{2}\right]^{-},\left[\mathrm{BF}\left(\mathrm{O}_{2} \mathrm{CCF}_{3}\right)_{3}\right]^{-},\left[\mathrm{B}\left(\mathrm{O}_{2} \mathrm{CCF}_{3}\right)_{4}\right]^{-}$, BODIPY-F-O $\mathrm{CCF}_{3}$, and BODIPY- $\left(\mathrm{O}_{2} \mathrm{CCF}_{3}\right)_{2}$, all show peaks between -1 and $1 \mathrm{ppm}$, thus fitting the experimental results, 3 days after the addition of TFA. Because addition of TFA causes broadening of the peaks, several boron-containing products likely exist in the NMR solutions of BODIPYs 1, 3, 4 , and 5 .

2.1.2. ${ }^{1} \mathrm{H} N M R$. The ${ }^{1} \mathrm{H}$ NMR spectra are shown in Figures S6-S20 of the Supporting Information. In the case of $4,4^{\prime}$ -
difluoro-BODIPY 1 , the characteristic singlets corresponding to the 1,7- and 3,5-methyl groups and the 2,6-hydrogen atoms changed $1 \mathrm{~h}$ after addition of TFA. This is consistent with the ${ }^{11} \mathrm{~B}$ spectra data suggesting stability of BODIPY 1 over a period of about $1 \mathrm{~h}$. On the other hand, the ${ }^{1} \mathrm{H}$ NMR data confirmed the stability of $4,4^{\prime}$-dicyano-BODIPY 2 with all characteristic peaks remaining singlets for up to 2 days and no changes in the chemical shifts. In the case of 4,4'-dimethyl-BODIPY 3, 4,4'diphenyl-BODIPY $\mathbf{4}$, and 4,4'-dimethoxy-BODIPY 5 all characteristic peaks disappeared immediately upon addition of 10 equiv of TFA, indicating the lower stability of these three BODIPYs relative to $\mathbf{1}$ and 2 . These results are consistent with the conclusions drawn from the ${ }^{11} \mathrm{~B}$ spectra with the exception of the 4,4'-diphenyl-BODIPY 4, which appears to be less stable than suggested by the ${ }^{11} \mathrm{~B}$ NMR results, as a result of the observed broadening of the ${ }^{11} \mathrm{~B}$ NMR signal.

Interestingly, for all investigated compounds, a new peak appears at around $10 \mathrm{ppm}$ for BODIPYs $\mathbf{1}, \mathbf{2}$, and $\mathbf{5}$ and around $11 \mathrm{ppm}$ for BODIPYs 3 and 4. This peak also appears in the spectrum of BODIPY 2, which otherwise remains unchanged. Analysis of computer-simulated ${ }^{1} \mathrm{H}$ NMR spectra of different possible compounds suggests that these peaks could be due to several possible species, including TFA (ca. $10 \mathrm{ppm}$ ), TFA dimer (calculated chemical shift of $13.8 \mathrm{ppm}$ ), and to a complex between protonated dipyrromethene $\left(\mathrm{DPMH}^{+}\right)$and TFA anion (calculated chemical shift of $13.0 \mathrm{ppm}$ ). The ${ }^{1} \mathrm{H}$ NMR spectra of TFA at $0.3,0.7,1.7$, and $3.3 \mathrm{M}$ concentrations in $\mathrm{CDCl}_{3}$ were also obtained experimentally and are shown in the Supporting Information, Figure S21.

In the case of BODIPY 2, the observed peak at ca. $11 \mathrm{pm}$ is most likely due to a hydrogen-bonded complex between $4,4^{\prime}$ dicyano-BODIPY 2 and TFA, as also observed in the ${ }^{11} \mathrm{~B}$ NMR spectrum (Table S2, Supporting Information). The calculated chemical shift for such a complex between $\mathbf{2}$ and one molecule of TFA is 12.1 ppm, whereas a complex of 2 with two molecules of TFA has a calculated chemical shift of $11.9 \mathrm{ppm}$. The energetics of formation of such complexes is analyzed in Sections 2.3 and 2.6 of this paper. An analogous complex between 4,4'-difluoro-BODIPY 1 and one TFA (calculated shift of $11.3 \mathrm{ppm}$ ) might be responsible for the observed peak for this compound. However, our calculations indicate that the hydrogen-bonded complexes for BODIPYs 4 (6.69 ppm) and 5 (16.7 ppm) do not generate peaks in this region. Therefore, because BODIPYs 3-5 decompose rapidly upon addition of TFA, the observed new downfield peaks for these compounds are likely due to the complex between protonated dipyrromethene $\left(\mathrm{DPMH}^{+}\right)$and TFA anion.

2.2. TLC. TLC analysis on silica gel plates was performed using dichloromethane/hexane 1:1 for elution, 3 days after the addition of TFA, following the ${ }^{11} \mathrm{~B}$ NMR experiments. A schematic representation of the results obtained is depicted in Figure S22 of the Supporting Information. For 4,4'-difluoroBODIPY 1, several spots were observed, most of higher polarity than the starting compound. One of the spots had the same $R_{\mathrm{f}}$ value $\left(R_{\mathrm{f}}=0.71\right)$ as 1 , and a co-spot with starting material indicated that it was starting BODIPY 1 . These results indicate that 1 partially decomposes in the presence of TFA, producing several products. On the other hand, the TLC plate for $4,4^{\prime}$ dicyano-BODIPY 2 showed only one single spot with the same $R_{\mathrm{f}}$ value as the original BODIPY $2\left(R_{\mathrm{f}}=0.11\right)$, indicating that this compound remained stable 3 days after addition of TFA, in agreement with the NMR results. In contrast, for 4,4'-dimethylBODIPY 3 and 4,4'-dimethoxy-BODIPY 5, no starting 
BODIPY was observed by TLC, indicating complete decomposition of the original compounds, into more polar products in the case of 3 and less polar products in the case of 5. For 4,4'-diphenyl-BODIPY 4 several spots were observed by TLC, one of them with the same $R_{\mathrm{f}}$ value as the starting BODIPY $\left(R_{\mathrm{f}}=0.84\right)$, indicating that some of 4 still remains 3 days after addition of TFA. These results are in agreement with the NMR data, also suggesting the unusually high stability of BODIPY 2. 4,4'-Difluoro-BODIPY 1 and 4,4'-diphenylBODIPY 4 demonstrate intermediate stability, whereas $4,4^{\prime}$ dimethyl-BODIPY 3 and 4,4'-dimethoxy-BODIPY $\mathbf{5}$ are the least stable of the series studied.

2.3. Absorption and Emission Studies. Fluorescence emission spectra (Figures S23-S27, Supporting Information) and normalized UV-vis absorption spectra (Figures S28-S32, Supporting Information) in tetrahydrofuran (THF) were obtained 3 days after the addition of 10 equiv of TFA. Excess amount of triethylamine was added to the samples to neutralize the excess TFA, and the neutralized spectra were also obtained. The 4,4'-dicyano-BODIPY 2 is the only compound, for which no obvious changes were observed in both the absorption and emission spectra. Only a slight decrease in emission intensity was observed, maybe as result of the formation of a BODIPY 2.*TFA complex (see below and Section 2.6), as suggested by the NMR experiments. In contrast, all other BODIPYs from the studied series showed pronounced fluorescence quenching and significant broadening of the main absorption band, along with slight change of the maximum absorption and emission wavelengths. Furthermore, a distinct absorption wavelength shift from ca. 500 to $450 \mathrm{~nm}$ was observed in the UV-vis spectra of 4,4'-dimethyl-BODIPY 3 and 4,4'-dimethoxyBODIPY $\mathbf{5}$ upon neutralization with excess amount of triethylamine, indicating the formation of dipyrromethene (DPM). For 4,4'-difluoro-BODIPY 1 and 4,4'-diphenylBODIPY 4, no such wavelength change was apparent. The characteristic BODIPY shoulder peak at $475 \mathrm{~nm}$ suggests that some 4,4'-difluoro-BODIPY 1 and 4,4'-diphenyl-BODIPY 4 remained unchanged, in agreement with the TLC results and NMR results.

The slight decrease in the fluorescence of 4,4'-dicyanoBODIPY 2 might be due to formation of a hydrogen-bonded complex between this compound and TFA. The existence of such complex was also suggested in the NMR sections above. To analyze this complex, we performed time-dependent (TD) M06-2X/6-31+G(d, p) calculations of BODIPY 2 and BODIPY $2 \cdots$ TFA. The results demonstrate that [BODIPY $2 \cdots$ TFA] is fluorescent with maximum absorption and emission wavelengths very similar to BODIPY 2 (431 vs $432 \mathrm{~nm}$ for absorption and 470 vs 472 for emission). The oscillator strengths are also similar but these parameters alone are not enough to predict the experimental quantum yields, which could depend on different de-excitation paths. The formation of this complex could explain the observed fluorescence even 3 days after the addition of TFA to BODIPY 2. As mentioned in the NMR section, this could also explain the slight shift in the ${ }^{11} \mathrm{~B}$ NMR spectrum for BODIPY 2 as well as the downfield peak observed in the ${ }^{1} \mathrm{H}$ NMR spectrum of $\mathbf{2}$ upon addition of TFA.

The calculated forms and energies of the frontier molecular orbitals of the studied BODIPYs are given in Figure S33, Supporting Information. As can be expected and in agreement with previous studies, substitution at the boron atom does not significantly change the character and the energies of the orbitals. ${ }^{36}$ The most pronounced difference is observed for cyano substitution: it stabilizes both the highest occupied molecular orbital (HOMO) and the lowest unoccupied molecular orbital by about $0.3-0.4 \mathrm{eV}$ when compared to 4,4'-difluoro-BODIPY 1. The lower HOMO level obtained for BODIPY 2 also suggests higher stability of this compound toward oxidation.

2.4. Mass Spectrometry Studies. High-resolution mass spectrometry (HRMS) (electrospray ionisation time-of-flight (ESI-TOF)) analysis was performed 3 days after the addition of 10 equiv of TFA to the series of BODIPY compounds (Figures S34-S38, Supporting Information). The peaks corresponding to $[\mathrm{M}+\mathrm{H}]^{+},[\mathrm{M}+\mathrm{Na}]^{+}$, and $[\mathrm{M}+\mathrm{K}]^{+}$for $4,4^{\prime}$-dicyano-BODIPY 2 , were clearly identified in the mass spectrum of this compound. This result further confirms the high stability of BODIPY 2 under these conditions. For 4,4'-difluoro-BODIPY 1, the $[\mathrm{M}+\mathrm{Na}]^{+}$peak was also identified, indicating that some of this BODIPY remains. However, for the $4,4^{\prime}$-dimethylBODIPY 3, 4,4'-diphenyl-BODIPY 4, and 4,4'-dimethoxyBODIPY 5, no molecular ion peak was observed. In these cases, only the peak corresponding to dipyrromethene (DPM) was found, suggesting that these compounds are unstable under TFA and/or the mass analysis conditions. These results are in agreement with the NMR, TLC, UV-vis, and fluorescence data above. It is possible that in the case of $4,4^{\prime}$-diphenyl-BODIPY 4, any remaining BODIPY decomposes during the mass analysis. Similarly, BODIPY-( $\left(\mathrm{OCOCF}_{3}\right)_{2}$, which is suggested by previous studies to be a product of the reaction between BODIPYs and TFA, ${ }^{33,34}$ was not identified from mass spectrometry analysis; it is possible that this compound decomposes during mass analysis as well.

2.5. Infrared (IR) Studies. The transmittance IR spectra were taken before and immediately after the addition of 2 equiv of TFA to $10 \mathrm{mM}$ BODIPY solutions in dichloromethane (Figures S39-S44, Supporting Information). The lower amount of TFA was chosen to reduce the rate of the reaction. For 4,4'-dimethyl-BODIPY 3 and 4,4' -dimethoxy-BODIPY 5, the fingerprint region changed dramatically, indicating that both compounds were unstable under these conditions, in agreement with the NMR data. As discussed in Section 2.1.1, these dramatic changes could be due to several possible species, including a complex between protonated dipyrromethene and TFA anion or TFA dimer. Upon addition of TFA to $4,4^{\prime}$ diphenyl-BODIPY 4, a new sharp peak around $1700 \mathrm{~cm}^{-1}$ appeared, which might be due to the formation of a complex or byproduct, as mentioned above. Nevertheless, the fingerprint area changed only slightly and good overlap was observed with the IR spectrum of the starting BODIPY 4 . In the case of $4,4^{\prime}$ difluoro-BODIPY 1 and 4,4'-dicyano-BODIPY 2, there was good overlap of the entire fingerprint region with the original IR spectra. According to the IR results, 4,4'-dimethyl-BODIPY 3 and 4,4'-dimethoxy-BODIPY 5 are unstable immediately upon addition of 2 equiv of TFA. The other three compounds remain stable, especially 4,4'-difluoro-BODIPY 1 and 4,4'dicyano-BODIPY 2, in agreement with the NMR results.

2.6. Computational Insights on Stability Trends. On the basis of ${ }^{1} \mathrm{H}$ NMR, MS, and UV-vis studies, we identified dipyrromethene (DPM) as a product of BODIPY decomposition reactions in the presence of excess TFA. Therefore, we investigated several parameters that evaluate the strength of the $\mathrm{B}-\mathrm{N}$ bond. Independently of the mechanisms of decomposition of BODIPYs, the conversion of BODIPY to DPM must involve breaking of the $\mathrm{B}-\mathrm{N}$ bond. The simplest measure 
Table 2. Calculated Molecular Parameters for the Series of BODIPYs Studied: B-N Bond Length $\left(r_{\mathrm{B}-\mathrm{N}}\right)$; B-N Symmetric Stretch Frequency $\left(\nu_{\mathrm{B}-\mathrm{N}}\right)$; Ionicity, Calculated as the Difference between B and N NPA Atomic Charges; Energy Necessary to Elongate the $\mathrm{B}-\mathrm{N}$ Bond by $0.2 \AA\left(\Delta E_{\mathrm{B}-\mathrm{N}}\right)$; Bond-Length Alternation Parameter (BLA) in the BODIPY Core; Substituent Mesomeric Parameters (M); Gibbs-Free Energy of the Hydrogen-Bond Formation between BODIPY and TFA in Chloroform $\left(\Delta G_{\text {BODIPY } \cdots \text { TFA }}\right)^{a}$

\begin{tabular}{|c|c|c|c|c|c|c|c|c|c|}
\hline 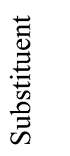 & 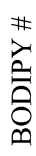 & 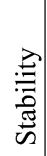 & $\begin{array}{l}r_{\mathrm{B}-\mathrm{N}} \\
(\AA)\end{array}$ & $\begin{array}{l}V_{\mathrm{B}-\mathrm{N}, \mathrm{sym}} \\
\left(\mathrm{cm}^{-1}\right)\end{array}$ & $\begin{array}{l}\text { Ionicity } \\
\text { (a.u.) }\end{array}$ & $\begin{array}{c}\Delta E_{\mathrm{B}-\mathrm{N}} \\
(\mathrm{kcal} / \mathrm{mol})\end{array}$ & $\begin{array}{l}B L A \\
(\AA)\end{array}$ & $M$ & $\begin{array}{c}\Delta G_{\text {BODIPY } \ldots \text { TFA }} \\
(\mathrm{kcal} / \mathrm{mol})\end{array}$ \\
\hline $\mathrm{CN}$ & 2 & 4 & 1.549 & 755 & 1.018 & 12.16 & 0.0345 & 0.66 & -3.71 \\
\hline $\mathrm{F}$ & 1 & & 1.554 & 787 & 1.876 & 12.09 & 0.0334 & 0.06 & -1.74 \\
\hline $\mathrm{Ph}$ & 4 & & 1.596 & 863 & 1.293 & 9.21 & 0.0410 & -0.01 & - \\
\hline $\mathrm{Me}$ & 3 & & 1.601 & 808 & 1.259 & 6.50 & 0.0392 & -0.18 & - \\
\hline $\mathrm{OMe}$ & 5 & | & 1.592 & 782 & 1.795 & 10.07 & 0.0366 & -0.56 & -6.93 \\
\hline
\end{tabular}

${ }^{a} \Delta E_{\mathrm{B}-\mathrm{N}}$ is calculated at CISD/aug-cc-pVDZ level in vacuum. All other parameters are calculated at B3LYP/6-31+G(d,p) level in chloroform.

of the strength of this bond is its length $\left(r_{\mathrm{B}-\mathrm{N}}\right.$ in Table 2$)$. As can be seen, with the exception of 4,4'-dimethoxy-BODIPY 5, the bond length is in good agreement with the trend of the observed stabilities under TFA: the shorter the $\mathrm{B}-\mathrm{N}$ bond, the higher the stability. This is in agreement with Groves et al., ${ }^{11}$ who used the $\mathrm{B}-\mathrm{N}$ bond length to explain different stabilities in 4,4'-difluoro-, diethyl-, and dimethoxy-BODIPYs. Their calculations showed that the dimethoxy-BODIPY had the longest $\mathrm{B}-\mathrm{N}$ bond length of the three compounds, whereas the difluoro had the shortest. Although their BODIPYs are different from our series, we observe similar behavior, with the difluoroBODIPY 1 displaying a shorter bond length than the dimethoxy-BODIPY 5. These authors also used the frequency of the symmetric $\mathrm{B}-\mathrm{N}$ stretching and the ionicity of the $\mathrm{B}-\mathrm{N}$ bond (calculated as the difference between the $\mathrm{B}$ and $\mathrm{N}$ atomic charges) to explain the observed stability. Our results are shown in Table 2. Although there does not seem to be a good correlation with the observed stability trend of the entire series, both of these parameters clearly indicate that the most stable $\mathrm{B}-\mathrm{N}$ bond is the one in 4,4'-dicyano-BODIPY 2 .

To get more insight into the strength of the $\mathrm{B}-\mathrm{N}$ bond, we calculated the energy necessary to elongate this bond by $0.2 \AA$. The value of $0.2 \AA$ was chosen arbitrarily, so it would not be too large but enough to demonstrate the differences in the bond strength. The data are presented in Table 2. The dicyano substitution results in slight strengthening of the $\mathrm{B}-\mathrm{N}$ bond compared with the 4,4'-difluoro-BODIPY 1, whereas methyl, phenyl, and methoxy substitutions destabilize the bond. With the exception of $4,4^{\prime}$-dimethoxy-BODIPY 5 , this trend does not contradict the observed stabilities; however, $\mathrm{CN}$ results in higher stability compared to F.

The reason for the greatest stability of the B-N bond in $4,4^{\prime}$ dicyano-BODIPY 2 might be better explained with the greatest aromaticity of the BODIPY core in this compound. The fact that 4,4'-dicyano substitution leads to smaller bond-length alternation (BLA) parameters and greater aromaticity was first noticed by Ortiz et al. ${ }^{17}$ In their paper, the authors used this parameter to explain the enhanced fluorescence and photostability of $4,4^{\prime}$-dicyano-substituted BODIPYs. In the present study, BLA demonstrates that 4,4'-dicyano-BODIPY 2 has the greatest aromaticity among the entire series studied. Thus, its $\mathrm{B}-\mathrm{N}$ bond is predicted to be the strongest. The BLA values are small but they are comparable and in agreement with Ortiz et al. ${ }^{17}$ The observed trend in BLA fits with experimentally observed stabilities, again, with the exception of $4,4^{\prime}$ dimethoxy-BODIPY $\mathbf{5}$.

In addition, the observed BODIPY stability correlates with the mesomeric effect of the substituents on boron: electronwithdrawing substituents on boron that decrease its charge lead to higher stability BODIPYs compared with electron-donating substituents. Comparison with the Hammett substituent mesomeric constants $(M)^{38}$ (Table 2) shows a correlation with the observed differences in stabilities. The strong electronwithdrawing $\mathrm{CN}$ group decreases the charge on boron leading to the most stable BODIPY, whereas the most electrondonating group $\mathrm{OMe}$ increases the boron charge and leads to the least stable BODIPY.

In their study, Rumyantsev et al. ${ }^{34,35}$ proposed a mechanism for the reaction of a series of 4,4'-difluoro-BODIPYs in the presence of different acids (TFA, TClA, $\mathrm{HCl}$, and $\mathrm{H}_{2} \mathrm{SO}_{4}$ ). It was suggested that the reaction begins with an attack of the acidic proton on the $\mathrm{F}$ atom and the formation of a hydrogenbonded complex [BODIPY $\cdots \mathrm{HA}$ ] followed by dissociation to form $[\mathrm{BODIPY} \cdots \mathrm{H}]^{+}$cation and $\mathrm{A}^{-}$(fast stage). In the second stage of the reaction (slow stage), the $\mathrm{B}-\mathrm{N}$ bond of the cation is broken to produce DPM and $\mathrm{BF}_{2}^{+}$. Finally, protonated DPM forms a $\left[\mathrm{DPMH}^{+} \ldots \mathrm{A}^{-}\right]$complex (fast stage). Our findings above are in agreement with Rumyantsev's second step of the reaction. The $\mathrm{B}-\mathrm{N}$ bond of $4,4^{\prime}$-dicyano-BODIPY 2 is the most stable among the studied BODIPYs making it the hardest to break.

The proposed first step of Rumyantsev's mechanism is in agreement with our analysis of possible hydrogen-bonded complexes between the BODIPYs and TFA. As discussed above, our NMR and fluorescence data for 4,4'-dicyanoBODIPY 2 indicate the formation of a [4,4'-dicyano-BODIPY $2 \cdots \mathrm{TFA}$ ] hydrogen-bonded complex immediately after the addition of TFA. Indeed, the formation of this hydrogen- 
bonded complex is energetically favorable $(\Delta G=-3.71)$, as can be seen from Table 2, where the Gibbs-free energy for the hydrogen-bond formation is calculated for BODIPYs 1, 2, and 5. Furthermore, we also calculated the Gibbs-free energy for the formation of a possible BODIPY $2 \cdots(\mathrm{TFA})_{2}$ complex, with each TFA hydrogen bound to the nitrogen atom of the $\mathrm{CN}$ group on the tetrahedral boron. The formation of such complex is not as favorable $(\Delta G=+10.84)$, and although the difference in energy is not large, the diTFA complex is less likely to be present, mostly due to the decrease of entropy.

On another hand, the effect of hydrogen bonding on the B$\mathrm{N}$ bond length is very interesting. The $\mathrm{B}-\mathrm{N}$ bond lengths decrease by about $0.02 \AA$ in all three compounds suggesting higher stability toward TFA addition. The HOMO level is also stabilized upon hydrogen bonding. This is especially pronounced for $-\mathrm{F}$ and $-\mathrm{CN}$ substitutions, where HOMO is lowered by around $0.3 \mathrm{eV}$. These two effects might explain the observed higher stabilities of 4,4'-dicyano-BODIPY 2 and 4,4'difluoro-BODIPY 1 compared with the other three BODIPYs on this series and also suggest higher stability toward oxidation.

In summary, on the basis of the presented calculated data and the observed ${ }^{11} \mathrm{~B}$ and ${ }^{1} \mathrm{H}$ NMR, MS, IR, UV-vis, and fluorescence spectra and TLC data, we believe that different BODIPYs most likely undergo different mechanisms of decomposition. For some of them, the mechanism might be the one described by Rumyantsev at al. ${ }^{34,35}$ For the other compounds, other mechanisms might predominate. Most importantly, for $4,4^{\prime}$-dicyano-BODIPY 2 , the reaction likely stops with the formation of an unusually stable hydrogenbonded complex with one molecule of TFA. These results could assist in the design and synthesis of BODIPYs that are stable under acidic solutions.

\section{CONCLUSIONS}

The stability of a series of 4,4' -disubstituted 1,3,5,7-tetramethyl8-phenyl-BODIPYs (-F, - CN, - Ph, - Me, and -OMe) toward addition of excess TFA, under air at room temperature, was studied systematically using several experimental techniques ( ${ }^{11} \mathrm{~B}$ NMR, ${ }^{1} \mathrm{H}$ NMR, MS, IR, TLC, UV-vis, and fluorescence) and computational modeling. In the absence of TFA, all BODIPYs in the series studied showed no decomposition after 3 days in $\mathrm{CDCl}_{3}$ or $\mathrm{CH}_{2} \mathrm{Cl}_{2}$ solutions under air. However, upon addition of excess TFA, all experimental methods $\left({ }^{11} \mathrm{~B}\right.$ and ${ }^{1} \mathrm{H}$ NMR, MS, IR, TLC, UV-vis, and fluorescence) indicated that 4,4'-dicyanoBODIPY 2 is the most stable compound, forming an unusually stable complex with TFA that remains unchanged for at least 3 days after addition of TFA. Several experimental techniques, including NMR, TLC, and IR, point toward the conclusion that 4,4'-difluoro-BODIPY 1 and 4,4'-diphenyl-BODIPY 4 exhibit intermediate stability, with some of the compounds remaining in the solution 3 days after addition of TFA. All methods also show that 4,4'-dimethyl-BODIPY 3 and 4,4'-dimethoxyBODIPY 5 are the least stable toward addition of TFA. Furthermore, mass spectrometry and UV-vis indicate that one product of decomposition of BODIPYs is dipyrromethene.

Comparison with the theoretically predicted ${ }^{11} \mathrm{~B}$ NMR and ${ }^{1} \mathrm{H}$ NMR shifts of different potential intermediates and products indicates that several possibilities exist. BODIPY$\left(\mathrm{OCOCF}_{3}\right)_{2}$, which is suggested by previous studies to be a product of the reaction between $4,4^{\prime}$-difluoro-BODIPYs and $\mathrm{TFA}^{33}$ is a potential intermediate, whereas boronium cations and $\mathrm{BR}_{2} \mathrm{TFA}$ do not appear to be formed by NMR.
The performed analysis and the comparison with theoretical calculations suggest that different BODIPYs from the series studied most likely follow different mechanisms of deboronation, especially 4,4'-dimethoxy-BODIPY 5. However, independently from the mechanism, the reaction involves breaking of the B-N bond to form DPM. Our results show that $4,4^{\prime}$ dicyano substitution stabilizes this bond, resulting in the most stable compound from the series studied. This is demonstrated by the $\mathrm{B}-\mathrm{N}$ bond length, by the energy necessary to elongate the $\mathrm{B}-\mathrm{N}$ bond, and by the bond-length alternation (BLA) parameter. BLA clearly indicates that $4,4^{\prime}$-dicyano-BODIPY 2 is the most aromatic among the studied compounds possessing the highest $\mathrm{B}-\mathrm{N}$ bond order. It is concluded that the strong electron-withdrawing substituent on boron $(-\mathrm{CN})$ leads to higher stability under the strong acidic conditions compared to electron-donating substituents. Our data suggest that the most stable BODIPY 2 likely exists in the form of a hydrogenbonded complex of unusual high stability after the addition of TFA.

The performed experimental and computational studies show that $4,4^{\prime}$-dicyano-BODIPY $\mathbf{2}$ is highly stable in TFA solution. This characteristic of this fluorescent dye is very important for its future applications, especially for its covalent attachment to peptides, antibodies, and other molecules for bioanalytic and bioimaging purposes.

\section{MATERIALS AND METHODS}

4.1. Instrumentation. All of the chemical reagents were purchased from VWR or Sigma-Aldrich and used without further purification. The reactions were monitored by thin layer chromatography (TLC) using precoated silica gel plates $(0.2$ $\mathrm{mm}$, polyester backed, $60 \AA$ ) and UV lamp. Column chromatography with silica gel (60 $\AA, 230-400$ mesh) and preparative TLC plates $\left(60 \AA, 20 \times 20 \mathrm{~cm}^{2}, 210-270 \mu \mathrm{m}\right)$ were used for the purifications. NMR spectra were collected using a Bruker AV-400 or AV-500 spectrometer at $300 \mathrm{~K}$ (operating at 400 or $500 \mathrm{MHz}$ for ${ }^{1} \mathrm{H}, 125 \mathrm{MHz}$ for ${ }^{13} \mathrm{C} \mathrm{NMR}$, and $128 \mathrm{MHz}$ for $\left.{ }^{11} \mathrm{~B} \mathrm{NMR}\right)$ in $\mathrm{CDCl}_{3}\left(7.26 \mathrm{ppm},{ }^{1} \mathrm{H}\right.$ and 77.0 $\mathrm{ppm},{ }^{13} \mathrm{C}$ ) and $\mathrm{BF}_{3} \cdot \mathrm{OEt}_{2}$ was set as reference $(0.00 \mathrm{ppm})$ for ${ }^{11} \mathrm{~B}$ NMR. Mass spectra were collected at the LSU Department of Chemistry Mass Spectrometry Facility using QSTAR XL ESI-TOF. The IR spectra were collected using a Bruker $\alpha$ spectrometer. All samples were scanned as dried ground samples by evaporating the solvents. BODIPYs $\mathbf{1},^{22-24} \mathbf{2 , 2 , 2 3 , 3 1}$ and $\mathbf{5}^{22,23,31}$ were prepared as previously reported in the literature.

4.2. Computational Methods. The geometries of all compounds and complexes were optimized without symmetry constraints using the B3LYP/6-31+G(d,p) level. ${ }^{39-41}$ The stationary points on the potential energy surface were confirmed with frequency calculations. The bond elongation energies were studied at the CISD/aug-cc-pVDZ level. ${ }^{42}$ The NMR chemical shifts were calculated at the B3LYP/6$31+G(d, p)$ level using the gauge-independent atomic orbital method. ${ }^{4-47}$ This method is suggested for effective but reliable NMR results. ${ }^{48,49}$ Our previous work on NMR spectra of BODIPYs has also demonstrated the reliability of this method. ${ }^{36}$ The atomic charges were calculated at the B3LYP/ $6-31+G(d, p)$ level using the NPA scheme. ${ }^{50-52}$ The absorption and emission spectra for BODIPY 2 and its hydrogen-bonded complex [BODIPY 2 $\cdots$ TFA] were calculated using the TDDFT method ${ }^{53}$ and the M06-2X/6-31+G(d,p) level, ${ }^{54}$ as recommended by Jacquemin at al. ${ }^{55,56}$ The solvent effects 
were taken into account using the polarized continuum model. ${ }^{57,58}$ The bond dissociation energies were calculated in vacuum. The optimized geometries, NMR shifts, NPA charges, and IR spectra were calculated in chloroform. The absorption and emission spectra of BODIPY 2 and [BODIPY $2 \cdots \mathrm{TFA}$ ] were calculated in THF. All calculations were performed using the Gaussian 09 program package. ${ }^{59}$

4.3. Synthesis of 4,4'-Dimethyl-1,3,5,7-tetramethyl-8phenyl-BODIPY 3. A solution of methylmagnesium bromide in diethyl ether $(0.46 \mathrm{~mL}, 3 \mathrm{M}, 1.39 \mathrm{mmol})$ was added to a solution of BODIPY $1(50.0 \mathrm{mg}, 0.154 \mathrm{mmol})$ in dry THF (10 $\mathrm{mL})$ at $0{ }^{\circ} \mathrm{C}$. After stirring in an ice bath for $10 \mathrm{~min}$, water $(2$ $\mathrm{mL}$ ) was added, and the resulting mixture was washed successively with distilled water $(10 \mathrm{~mL})$ and brine $(10 \mathrm{~mL})$. The organic layer was dried over anhydrous $\mathrm{Na}_{2} \mathrm{SO}_{4}$ and concentrated under reduced pressure. The residue was purified by preparative TLC using 50\% dichloromethane/hexane for elution and recrystallized from dichloromethane/hexane to give the title BODIPY (38.2 mg, 78\%) as a red solid. ${ }^{1} \mathrm{H}$ NMR (500 $\left.\mathrm{MHz}, \mathrm{CDCl}_{3}\right) \delta 7.49-7.42(\mathrm{~m}, 3 \mathrm{H}), 7.31(\mathrm{dd}, J=7.5,1.7 \mathrm{~Hz}$, $2 \mathrm{H}), 5.98(\mathrm{~s}, 2 \mathrm{H}), 2.49(\mathrm{~s}, 6 \mathrm{H}), 1.35(\mathrm{~s}, 6 \mathrm{H}), 0.27(\mathrm{~s}, 6 \mathrm{H}) .{ }^{13} \mathrm{C}$ NMR $\left(125 \mathrm{MHz}, \mathrm{CDCl}_{3}\right) \delta 152.14,142.10,138.95,136.43$, $129.81,128.83,128.43,127.53,121.56,16.45,14.58 .{ }^{11} \mathrm{~B}$ NMR $\left(128 \mathrm{MHz}, \mathrm{CDCl}_{3}\right) \delta-0.78(\mathrm{~s})$. HRMS (ESI-TOF) $\mathrm{m} / z$ 317.2198 $[\mathrm{M}+\mathrm{H}]^{+}$, calcd for $\mathrm{C}_{21} \mathrm{H}_{26} \mathrm{BN}_{2}$ 317.2187. Melting point: $118-123{ }^{\circ} \mathrm{C}$.

4.4. Synthesis of 4,4'-Diphenyl-1,3,5,7-tetramethyl-8phenyl-BODIPY 4. BODIPY 1 (20.0 mg, $0.06 \mathrm{mmol})$ was dissolved in anhydrous THF $(3 \mathrm{~mL})$, and the solution was cooled in dry ice. Phenylmagnesium bromide in diethyl ether $(0.18 \mathrm{~mL}, 3 \mathrm{M}, 0.56 \mathrm{mmol})$ was added, and the resulting solution was left to stir overnight. When the reaction was complete, water $(10 \mathrm{~mL})$ was added, and the aqueous layer was separated and extracted with diethyl ether $(2 \times 20 \mathrm{~mL})$. The combined organic extracts were dried over anhydrous $\mathrm{Na}_{2} \mathrm{SO}_{4}$ and concentrated under reduced pressure. The residue was purified by preparative TLC using 50\% dichloromethane/ hexane for elution and recrystallized from dichloromethane/ hexane to give the title BODIPY $(14.7 \mathrm{mg}, 54 \%)$ as a dark orange solid. ${ }^{1} \mathrm{H}$ NMR $\left(400 \mathrm{MHz}, \mathrm{CDCl}_{3}\right) \delta 7.50-7.19(\mathrm{~m}$, $15 \mathrm{H}), 5.93(\mathrm{~s}, 2 \mathrm{H}), 1.80(\mathrm{~s}, 6 \mathrm{H}), 1.41(\mathrm{~s}, 6 \mathrm{H}) .{ }^{13} \mathrm{C}$ NMR $(125$ $\mathrm{MHz} \mathrm{CDCl}_{3}$ ) $\delta 154.59,142.38,140.23,136.05,133.83,131.44$, $128.84,128.53,127.20,125.66,121.97,16.98,14.73 .{ }^{11} \mathrm{~B}$ NMR $\left(128 \mathrm{MHz}, \mathrm{CDCl}_{3}\right) \delta-0.10$ (s). HRMS (ESI-TOF) $\mathrm{m} / z$ 441.2506 $[\mathrm{M}+\mathrm{H}]^{+}$, calcd for $\mathrm{C}_{31} \mathrm{H}_{30} \mathrm{BN}_{2}$ 441.2502. Melting point: $125-130{ }^{\circ} \mathrm{C}$.

4.5. Stability Study of BODIPY Analogs in the Presence of Excess TFA. Ten equivalents of TFA ( $1 \mathrm{M}$ in deuterated chloroform) were added to each BODIPY solution at a concentration of $30-80 \mathrm{mM}$, prepared by dissolving 5-13 $\mathrm{mg}(0.014-0.032 \mathrm{mmol})$ in deuterated chloroform $(0.4 \mathrm{~mL})$ in an NMR tube. After TFA addition, the NMR tube was shook to mix the reagents. ${ }^{1} \mathrm{H}$ NMR and ${ }^{11} \mathrm{~B}$ NMR spectra were obtained at time intervals up to 2 and 3 days. After 3 days, the samples were also analyzed by MS (ESI-TOF), TLC, UV-vis, and fluorescence spectrometry. The IR spectra were obtained on $10 \mathrm{mM}$ solutions of BODIPYs $\mathbf{1 - 5}$ in dichloromethane, immediately after addition of 2 equiv of TFA (100 mM in dichloromethane).

\section{ASSOCIATED CONTENT}

\section{Supporting Information}

The Supporting Information is available free of charge on the ACS Publications website at DOI: 10.1021/acsomega.8b00404.

${ }^{11} \mathrm{~B}$ and ${ }^{1} \mathrm{H}$ NMR spectra; calculated chemical shifts; TLC studies; fluorescence and UV-vis spectra; frontier orbitals; mass spectra; IR spectra; Cartesian coordinates for the optimized in chloroform ground states of BODIPY 1-5 and the hydrogen-bonded complex to TFA, and the optimized in THF excited states of BODIPY 2 and its hydrogen-bonded complex to TFA (PDF)

\section{AUTHOR INFORMATION}

\section{Corresponding Author}

*E-mail: petia.bobadova@rockhurst.edu.

ORCID

M. Graça H. Vicente: 0000-0002-4429-7868

Petia Bobadova-Parvanova: 0000-0002-1965-419X

\section{Notes}

The authors declare no competing financial interest.

\section{ACKNOWLEDGMENTS}

This work was supported by the National Science Foundation (CHE-1362641). The authors are thankful to the Louisiana Optical Network Initiative (www.loni.org) for the use of their computer facilities.

\section{REFERENCES}

(1) Loudet, A.; Burgess, K. Bodipy Dyes and Their Derivatives: Syntheses and Spectroscopic Properties. Chem. Rev. 2007, 107, 48914932.

(2) Marfin, Y. S.; Solomonov, A. V.; Timin, A. S.; Rumyantsev, E. V. Recent Advances of Individual Bodipy and Bodipy-Based Functional Materials in Medical Diagnostics and Treatment. Curr. Med. Chem. 2017, 24, 2745-2772.

(3) Uppal, T.; Bhupathiraju, N. V. S. D. K.; Vicente, M. G. H. Synthesis and Cellular Properties of Near-IR Bodipy-Peg and Carbohydrate Conjugates. Tetrahedron 2013, 69, 4687-4693.

(4) Joshi, B. P.; Wang, T. D. Exogenous Molecular Probes for Targeted Imaging in Cancer: Focus on Multi-Modal Imaging. Cancers 2010, 2, 1251-1287.

(5) Ulrich, G.; Ziessel, R.; Harriman, A. The Chemistry of Fluorescent Bodipy Dyes: Versatility Unsurpassed. Angew. Chem., Int. Ed. 2008, 47, 1184-1201.

(6) Boens, N.; Leen, V.; Dehaen, W. Fluorescent Indicators Based on Bodipy. Chem. Soc. Rev. 2012, 41, 1130-1172.

(7) Kamkaew, A.; Lim, S. H.; Lee, H. B.; Kiew, L. V.; Chung, L. Y.; Burgess, K. Bodipy Dyes in Photodynamic Therapy. Chem. Soc. Rev. 2013, 42, 77-88.

(8) Boens, N.; Verbelen, B.; Dehaen, W. Postfunctionalization of the Bodipy Core: Synthesis and Spectroscopy. Eur. J. Org. Chem. 2015, 2015, 6577-6595.

(9) Kowada, T.; Maeda, H.; Kikuchi, K. Bodipy-Based Probes for the Fluorescence Imaging of Biomolecules in Living Cells. Chem. Soc. Rev. 2015, 44, 4953-4972.

(10) Lundrigan, T.; Crawford, S. M.; Cameron, T. S.; Thompson, A. Cl-Bodipys: A Bodipy Class Enabling Facile B-Substitution. Chem. Comm. 2012, 48, 1003-1005.

(11) Groves, B. R.; Crawford, S. M.; Lundrigan, T.; Matta, C. F.; Sowlati-Hashjin, S.; Thompson, A. Synthesis and Characterisation of the Unsubstituted Dipyrrin and 4,4-Dichloro-4-Bora-3a,4a-Diaza-SIndacene: Improved Synthesis and Functionalisation of the Simplest Bodipy Framework. Chem. Comm. 2013, 49, 816-818. 
(12) Lundrigan, T.; Thompson, A. Conversion of F-Bodipys to ClBodipys: Enhancing the Reactivity of F-Bodipys. J. Org. Chem. 2013, 78, 757-761.

(13) Lundrigan, T.; Cameron, T. S.; Thompson, A. Activation and Deprotection of F-Bodipys Using Boron Trihalides. Chem. Comm. 2014, 50, 7028-7031.

(14) Bonnier, C.; Piers, W. E.; Parvez, M. Isomeric Dipyrrinato and Dipyrromethanato Boranes. Organometallics 2011, 30, 1067-1072.

(15) Heyer, E.; Ziessel, R. Step-by-Step Synthesis of Multimodule Assemblies Engineered from Bodipy, Dpp, and Triphenylamine Moieties. J. Org. Chem. 2015, 80, 6737-6753.

(16) Yamane, H.; Tanaka, K.; Chujo, Y. Simple and Valid Strategy for the Enhancement of the Solid-Emissive Property of Boron Dipyrromethenes. Tetrahedron Lett. 2015, 56, 6786-6790.

(17) Duran-Sampedro, G.; Esnal, I.; Agarrabeitia, A. R.; Banuelos Prieto, J.; Cerdan, L.; Garcia-Moreno, I.; Costela, A.; Lopez-Arbeloa, I.; Ortiz, M. J. First Highly Efficient and Photostable E and C Derivatives of 4,4-Difluoro-4-Bora-3a,4a-Diaza-S-Indacene (Bodipy) as Dye Lasers in the Liquid Phase, Thin Films, and Solid-State Rods. Chem. - Eur. J. 2014, 20, 2646-2653.

(18) Higham, L.; Davies, L.; Wallis, J.; Probert, M. Efficient Multigram Syntheses of Air-Stable, Fluorescent Primary Phosphines Via Palladium-Catalyzed Phosphonylation of Aryl Bromides. Synthesis 2014, 46, 2622-2628.

(19) Lu, J.-S.; Ko, S.; Walters, N. R.; Wang, S. Decorating Bodipy with Three- and Four-Coordinate Boron Groups. Org. Lett. 2012, 14, $5660-5663$.

(20) Davies, L. H.; Harrington, R. W.; Clegg, W.; Higham, L. J. Br2bodpr2: Highly Fluorescent Alternatives to PPh3 and Phpcy2. Dalton Trans. 2014, 43, 13485-13499.

(21) Liu, K.-M.; Tsai, M.; Jan, M.; Chau, C.; Wang, W. Convenient One-Pot Procedure for Synthesizing 4,4'-Dimethoxy-Boradiaza-SIndacene Dyes and Their Application to Cell Labeling. Tetrahedron 2011, 67, 7919-7922.

(22) Richards, V. J.; Gower, A. L.; Smith, J. E. H. B.; Davies, E. S.; Lahaye, D.; Slater, A. G.; Lewis, W.; Blake, A. J.; Champness, N. R.; Kays, D. L. Synthesis and Characterisation of Bodipy Radical Anions. Chem. Comm. 2012, 48, 1751-1753.

(23) Wang, H.; Vicente, M. G.; Fronczek, F. R; Smith, K. M. Synthesis and Transformations of 5-Chloro-2,2'-Dipyrrins and Their Boron Complexes, 8-Chloro-Bodipys. Chem. - Eur. J. 2014, 20, 50645074.

(24) Nguyen, A. L.; Bobadova-Parvanova, P.; Hopfinger, M.; Fronczek, F. R.; Smith, K. M.; Vicente, M. G. H. Synthesis and Reactivity of 4,4-Dialkoxy-Bodipys: An Experimental and Computational Study. Inorg. Chem. 2015, 54, 3228-3236.

(25) Deschamps, J.; Chang, Y.; Langlois, A.; Desbois, N.; Gros, C. P.; Harvey, P. D. The First Example of Cofacial Bis(Dipyrrins). New J. Chem. 2016, 40, 5835-5845.

(26) Liu, B.; Novikova, N.; Simpson, M. C.; Timmer, M. S.; Stocker, B. L.; Sohnel, T.; Ware, D. C.; Brothers, P. J. Lighting up Sugars: Fluorescent Bodipy-Gluco-Furanose and -Septanose Conjugates Linked by Direct B-O-C Bonds. Org. Biomol. Chem. 2016, 14, 5205-5209.

(27) Tahtaoui, C.; Thomas, C.; Rohmer, F.; Klotz, P.; Duportail, G.; Mély, Y.; Bonnet, D.; Hibert, M. Convenient Method to Access New 4,4-Dialkoxy- and 4,4-Diaryloxy-Diaza-S-Indacene Dyes: Synthesis and Spectroscopic Evaluation. J. Org. Chem. 2007, 72, 269-272.

(28) Smithen, D. A.; Baker, A. E.; Offman, M.; Crawford, S. M.; Cameron, T. S.; Thompson, A. Use of F-Bodipys as a Protection Strategy for Dipyrrins: Optimization of $\mathrm{BF}_{2}$ Removal. J. Org. Chem. 2012, 77, 3439-3453.

(29) Zhao, N.; Xuan, S.; Byrd, B.; Fronczek, F. R.; Smith, K. M.; Vicente, M. G. Synthesis and Regioselective Functionalization of Perhalogenated Bodipys. Org. Biomol. Chem. 2016, 14, 6184-6188.

(30) Cieślik-Boczula, K.; Burgess, K.; Li, L.; Nguyen, B.; Pandey, L.; De Borggraeve, W. M.; Van der Auweraer, M.; Boens, N. Photophysics and Stability of Cyano-Substituted Boradiazaindacene Dyes. Photochem. Photobiol. Sci. 2009, 8, 1006-1015.
(31) Li, L.; Nguyen, B.; Burgess, K. Functionalization of the 4,4Difluoro-4-Bora-3a,4a-Diaza-S-Indacene (Bodipy) Core. Bioorg. Med. Chem. Lett. 2008, 18, 3112-3116.

(32) Niu, L.; et al. Colorimetric Sensors with Different Reactivity for the Quantitative Determination of Cysteine, Homocysteine and Glutathione in a Mixture. RSC Adv. 2015, 5, 13042-13045.

(33) Yang, L.; Simionescu, R.; Lough, A.; Yan, H. Some Observations Relating to the Stability of the Bodipy Fluorophore under Acidic and Basic Conditions. Dyes Pigm. 2011, 91, 264-267.

(34) Rumyantsev, E. V.; Alyoshin, S. N.; Marfin, Y. S. Kinetic Study of Bodipy Resistance to Acids and Alkalis: Stability Ranges in Aqueous and Non-Aqueous Solutions. Inorg. Chim. Acta 2013, 408, 181-185.

(35) Rumyantsev, E. V.; Aleshin, S. N.; Desoki, A.; Marfin, Y. S.; Antina, E. V. Kinetic Resistance of Borofluoride Complexes of Dipyrrolylmethenes to Acids. Russ. J. Inorg. Chem. 2013, 58, 596-601.

(36) Nguyen, A. L.; Wang, M.; Bobadova-Parvanova, P.; Do, Q.; Zhou, Z.; Fronczek, F. R.; Smith, K. M.; Vicente, M. G. H. Synthesis and Properties of B-Cyano-Bodipys. J. Porphyrins Phthalocyanines 2016, 20, 1409-1419.

(37) Duran-Sampedro, G.; Agarrabeitia, A. R.; Garcia-Moreno, I.; Costela, A.; Bañuelos, J.; Arbeloa, T.; López Arbeloa, I.; Chiara, J. L.; Ortiz, M. J. Chlorinated Bodipys: Surprisingly Efficient and Highly Photostable Laser Dyes. Eur. J. Org. Chem. 2012, 2012, 6335-6350.

(38) Hansch, C.; Leo, A.; Taft, R. W. A Survey of Hammett Substituent Constants and Resonance and Field Parameters. Chem. Rev. 1991, 91, 165-195.

(39) Becke, A. D. Becke's Three Parameter Hybrid Method Using the Lyp Correlation Functional. J. Chem. Phys. 1993, 98, 5648-5652.

(40) Lee, C.; Yang, W.; Parr, R. G. Development of the Colle-Salvetti Correlation-Energy Formula into a Functional of the Electron Density. Phys. Rev. B 1988, 37, 785.

(41) Ditchfield, R.; Hehre, W. J.; Pople, J. A. Self-Consistent Molecular-Orbital Methods. Ix. An Extended Gaussian-Type Basis for Molecular-Orbital Studies of Organic Molecules. J. Chem. Phys. 1971, 54, 724-728.

(42) Dunning, T. H., Jr. Gaussian Basis Sets for Use in Correlated Molecular Calculations. I. The Atoms Boron through Neon and Hydrogen. J. Chem. Phys. 1989, 90, 1007-1023.

(43) London, F. Théorie Quantique Des Courants Interatomiques Dans Les Combinaisons Aromatiques. J. Phys. Radium 1937, 8, 397409.

(44) McWeeny, R. Perturbation Theory for the Fock-Dirac Density Matrix. Phys. Rev. 1962, 126, 1028.

(45) Ditchfield, R. Self-Consistent Perturbation Theory of Diamagnetism: I. A Gauge-Invariant Lcao Method for Nmr Chemical Shifts. Mol. Phys. 1974, 27, 789-807.

(46) Wolinski, K.; Hinton, J. F.; Pulay, P. Efficient Implementation of the Gauge-Independent Atomic Orbital Method for Nmr Chemical Shift Calculations. J. Am. Chem. Soc. 1990, 112, 8251-8260.

(47) Cheeseman, J. R.; Trucks, G. W.; Keith, T. A.; Frisch, M. J. A Comparison of Models for Calculating Nuclear Magnetic Resonance Shielding Tensors. J. Chem. Phys. 1996, 104, 5497-5509.

(48) Lodewyk, M. W.; Siebert, M. R.; Tantillo, D. J. Computational Prediction of ${ }^{1} \mathrm{H}$ and ${ }^{13} \mathrm{C}$ Chemical Shifts: A Useful Tool for Natural Product, Mechanistic, and Synthetic Organic Chemistry. Chem. Rev. 2012, 112, 1839-1862.

(49) Jain, R.; Bally, T.; Rablen, P. R. Quantum-Chemical Simulation of ${ }^{1} \mathrm{H}$ NMR Spectra. 2. Comparison of DFT-Based Procedures for Computing Proton-Proton Coupling Constants in Organic Molecules. J. Org. Chem. 2009, 74, 4017-4023.

(50) Foster, J.; Weinhold, F. Natural Hybrid Orbitals. J. Am. Chem. Soc. 1980, 102, 7211-7218.

(51) Reed, A. E.; Weinstock, R. B.; Weinhold, F. Natural Population Analysis. J. Chem. Phys. 1985, 83, 735-746.

(52) Reed, A. E.; Curtiss, L. A.; Weinhold, F. Intermolecular Interactions from a Natural Bond Orbital, Donor-Acceptor Viewpoint. Chem. Rev. 1988, 88, 899-926. 
(53) Bauernschmitt, R.; Ahlrichs, R. Treatment of Electronic Excitations within the Adiabatic Approximation of Time Dependent Density Functional Theory. Chem. Phys. Lett. 1996, 256, 454-464.

(54) Zhao, Y.; Truhlar, D. G. The M06 Suite of Density Functionals for Main Group Thermochemistry, Thermochemical Kinetics, Noncovalent Interactions, Excited States, and Transition Elements: Two New Functionals and Systematic Testing of Four M06-class Functionals and 12 other Functionals. Theor. Chem. Acc. 2007, 120, 215-241.

(55) Laurent, A. D.; Adamo, C.; Jacquemin, D. Dye Chemistry with Time-Dependent Density Functional Theory. Phys. Chem. Chem. Phys. 2014, 16, 14334-14356.

(56) Le Guennic, B.; Jacquemin, D. Taking up the Cyanine Challenge with Quantum Tools. Acc. Chem. Res. 2015, 48, 530-537.

(57) Miertuš, S.; Scrocco, E.; Tomasi, J. Electrostatic Interaction of a Solute with a Continuum. A Direct Utilizaion of Ab Initio Molecular Potentials for the Prevision of Solvent Effects. Chem. Phys. 1981, 55, 117-129.

(58) Tomasi, J.; Mennucci, B.; Cammi, R. Quantum Mechanical Continuum Solvation Models. Chem. Rev. 2005, 105, 2999-3094.

(59) Frisch, M. J.; Trucks, G. W.; Schlegel, H. B.; Scuseria, G. E.; Robb, M. A.; Cheeseman, J. R.; Scalmani, G.; Barone, V.; Mennucci, B.; Petersson, G. A. et al. Gaussian 09, revision A.02; Gaussian, Inc.: Wallingford, CT, 2009. 